\title{
The ectopic posterior pituitary - a short case series
}

\section{Modi, MB BCh, FCRad (D), MMed}

Division of Radiology, Department of Radiation Sciences, Chris Hani Baragwanath Hospital, University of the Witwatersrand, Johannesburg

\begin{abstract}
The ectopic posterior pituitary (EPP) refers to the anomalous position of the distinctly bright posterior pituitary gland. Defective neural migration during embryogenesis is believed to be the cause of the EPP. This hypothesis is supported by the co-existence of other midline structural malformations where anterior pituitary hypoplasia and EPP are the end products.

The EPP often occurs in patients with growth hormone deficiency (GHD), and is a marker of GHD. The EPP should alert the radiologist to perform a precise MRI study as it is an important marker of anterior pituitary structure and function. The hyperintense posterior pituitary is due to its phospholipid content.
\end{abstract}

\section{Introduction}

The anterior and the posterior pituitary are composed of tissues that are embryologically and histologically different, as the normal pituitary development results from the upward growth of Rathke's pouch (ectoderm) fusing with the downward growth of the neuroectoderm of the diencephalon. ${ }^{1}$

Therefore defective neural migration during embryogenesis is believed to be the cause of the ectopic posterior pituitary (EPP). This may be partial or complete, possibly explaining why the EPP can be located at different sites of the stalk. ${ }^{2}$ This abnormal embryonic development hypothesis is supported by the co-existence of other midline structural malformations where anterior pituitary hypoplasia and EPP are the end products. ${ }^{2,3}$ The associated described midline anomalies include septo-optic dysplasia, grey matter hetrotopia, scizencephaly, corpus callosal dysgenesis, and anopthalmia. ${ }^{3}$

Another (less popular) hypothesis postulated for the EPP is transection of the cord possibly on the basis of prenatal or perinatal trauma. ${ }^{1}$

The pituitary functions as an endocrine gland under the control of the hypothalamus via the infundibular stalk. ${ }^{1}$

The EPP often occurs in patients with growth hormone deficiency (GHD), and is a marker of GHD. ${ }^{3}$ The cause of GHD maybe 'idiopathic' or can occur secondary to surgery, tumour or radiation. ${ }^{1}$ Idiopathic GHD may occur in isolation or in association with multiple anterior pituitary hormone deficiencies (MPHD). ${ }^{1}$ MPHD is defined as GHD associated with at least one other abnormality of the anterior pituitary hormones (including thyroid-stimulating hormone (TSH), adrenocorticotrophic hormone (ACTH), and prolactin). ${ }^{2}$ Previous studies have demonstrated that patients with a low growth hormone $(\mathrm{GH})$ level $(<3$ g/l) are more likely to have an EPP. ${ }^{1}$

\section{Magnetic resonance imaging (MRI) is the cornerstone for evaluat-} ing the hypothalamic-pituitary axis in children. ${ }^{4}$ It was instrumental in identifying the hyperintense EPP on T1 imaging, and has been found to be a significant contributing factor to the diagnosis of 'idiopathic' and permanent GHD. ${ }^{5}$ The distinctly hyperintense posterior pituitary is due to its phospholipid content. Imaging of the pituitary varies with age, and the adenohypophysis also demonstrates a high signal in the first 2 months of life. ${ }^{4}$ The pituitary gland height decreases during the first year of life, then increases to achieve its plateau after puberty. ${ }^{4}$ The magnetisation transfer ratio (MTR) has been noted to increase in males and females up to the age of $30 .{ }^{4}$ Dynamic contrast-enhanced studies have demonstrated simultaneous enhancement of the posterior pituitary lobe and the straight sinus, with the adenohypophysis enhancing slightly later - but all within 30 seconds. ${ }^{4}$

Although MRI provides excellent definition of the hypothalamicpituitary axis, gadolinium is necessary for better description of the stalk - especially if the stalk is thin. ${ }^{2}$ Adequate visualisation of the stalk is very important as it has a bearing on the adenohypophysis structure. Chen $e t a l .^{2}$ demonstrated that when the pituitary stalk was present, the adenohypophysis was only hypoplastic in $50 \%$ of cases, however when the pituitary stalk was 'absent' the adenohypophysis was hypoplastic in the vast majority of cases. This observation is very pertinent as it has therapeutic and prognostic implications. The visible pituitary stalk may be a sensitive marker for idiopathic GHD in EPP, whereas its absence constitutes a predictive factor for MPHD. ${ }^{2}$

Moreover, major technical strides achieved in DNA technology have assisted in shedding new light on the genetic causes of hypopituitarism and the EPP. ${ }^{3,5}$ Two gene abnormalities associated with hypopituitarism have been identified, GH-N encoding for GH and the GHRH receptor (GHRH-R); and thus far only the HESX1 gene appears to be linked to the $\mathrm{EPP}^{3,5}$

\section{Patients and methods}

We scanned 3 patients with EPP glands at the Chris Hani Baragwanath Hospital MR Department over a period of 3 months. The MR scans were done on a 1.5 tesla General Electric (GE) Signa Excite machine. Dynamic contrast injection was used in patients 2 and 3. A hand injection was used in patient 1 due to his tender age.

\section{Patient 1}

The patient was 5 weeks old, and was being investigated for MPHD. The sagittal T1-weighted MRI scan demonstrated the high signal intensity posterior pituitary lobe outside the confines of the sella, and a hypoplastic adenohypophysis. The stalk was not visualised pre or post intravenous contrast (Fig. 1).

\section{Patient 2}

The patient was 3 years old, and was a known patient with hypopituitarism on replacement therapy. The sagittal T1-weighted MRI scan demonstrated the high signal intensity posterior pituitary lobe outside the 


\section{CASE SERIES}

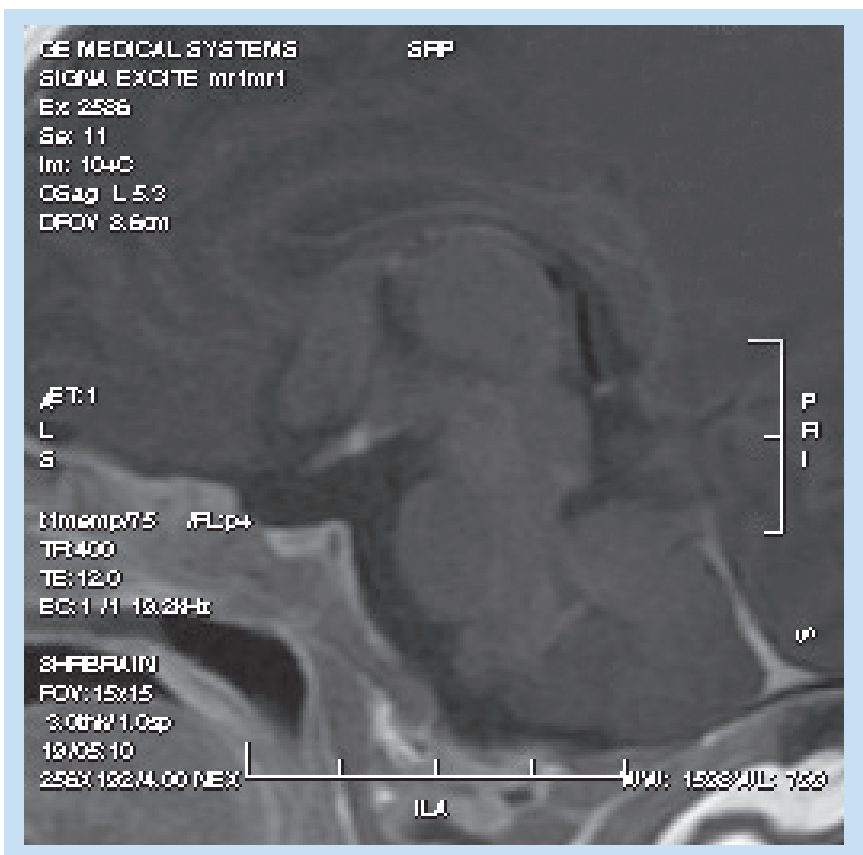

Fig. 1. A sagittal T1W post-contrast MRI scan demonstrating the hyperintense posterior pituitary gland outside the sella and along the pathway of the stalk. The pituitary stalk is not visualised. The adenohypophsis is hypoplastic.

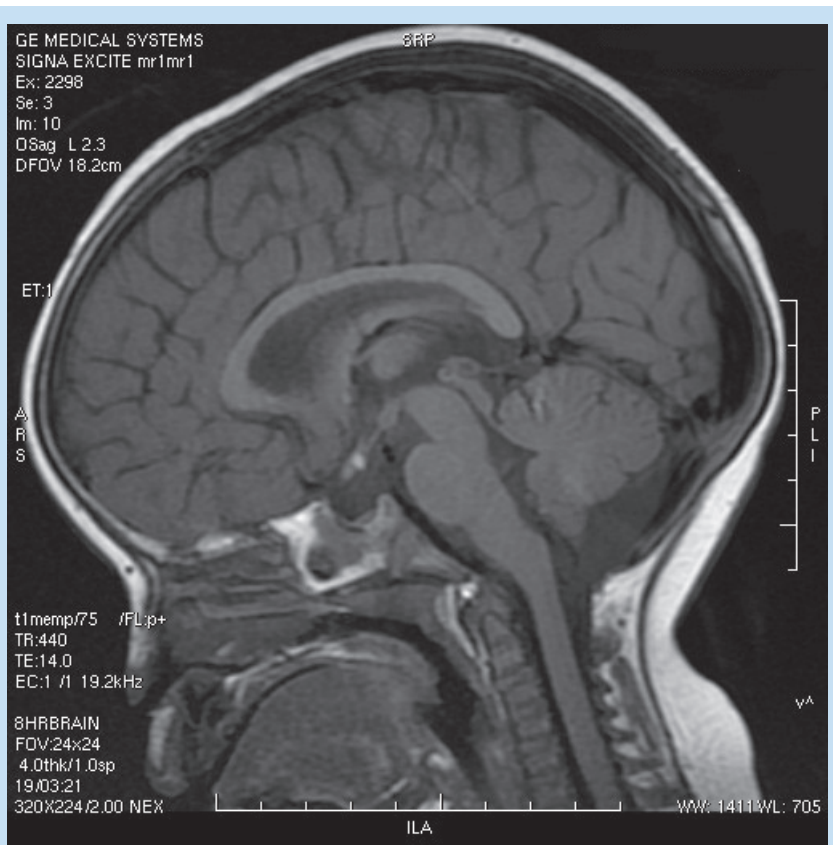

Fig. 2. A sagittal T1W MRI scan demonstrating the hyperintense posterior pituitary gland outside the sella, and adjacent to the optic chiasm. The pituitary stalk is not visualised. The adenohypophsis is hypoplastic. Note the small cyst in the pineal gland.

confines of the sella, a hypoplastic adenohypophysis, and again the stalk was not visualised pre or post intravenous contrast (Fig. 2).

\section{Patient 3}

The patient was a fully functional woman of 25 years (she worked as a cashier at the local supermarket). She appeared to be of shorter than average stature, was well spoken, and did not suffer from any seizure disorder.

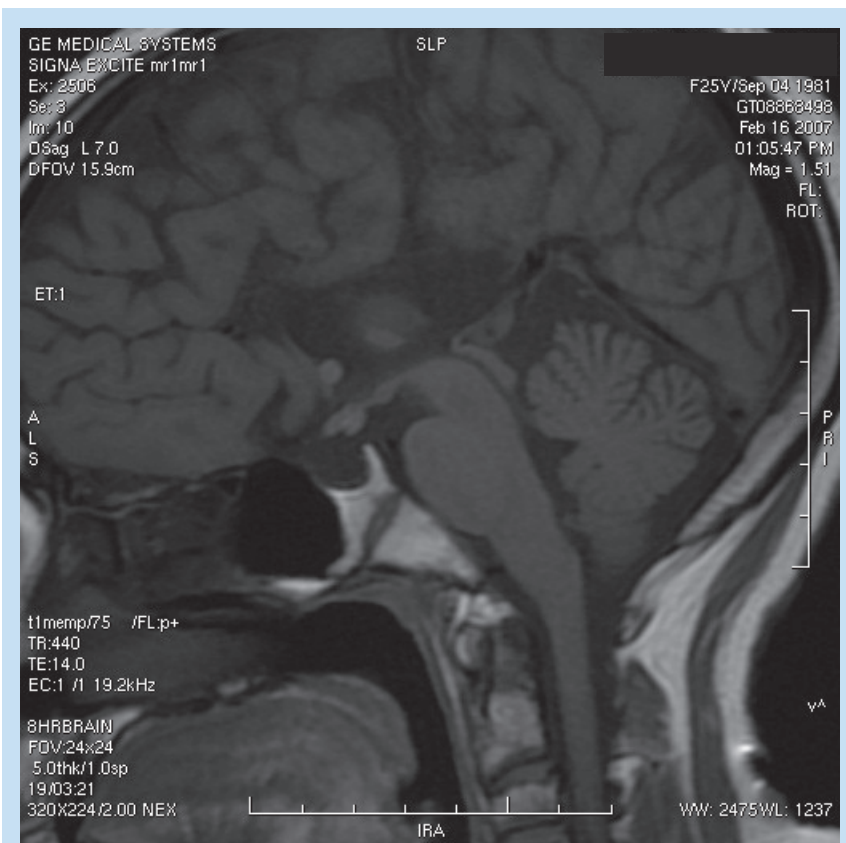

Fig. 3a. A sagittal T1W MRI scan demonstrating the not very hyperintense posterior pituitary gland outside the sella and along the pathway of the stalk, non-visualisation of the adenohypophsis, an absent corpus callosum, prominence of the anterior commisure, and a tiny cyst in the pineal.

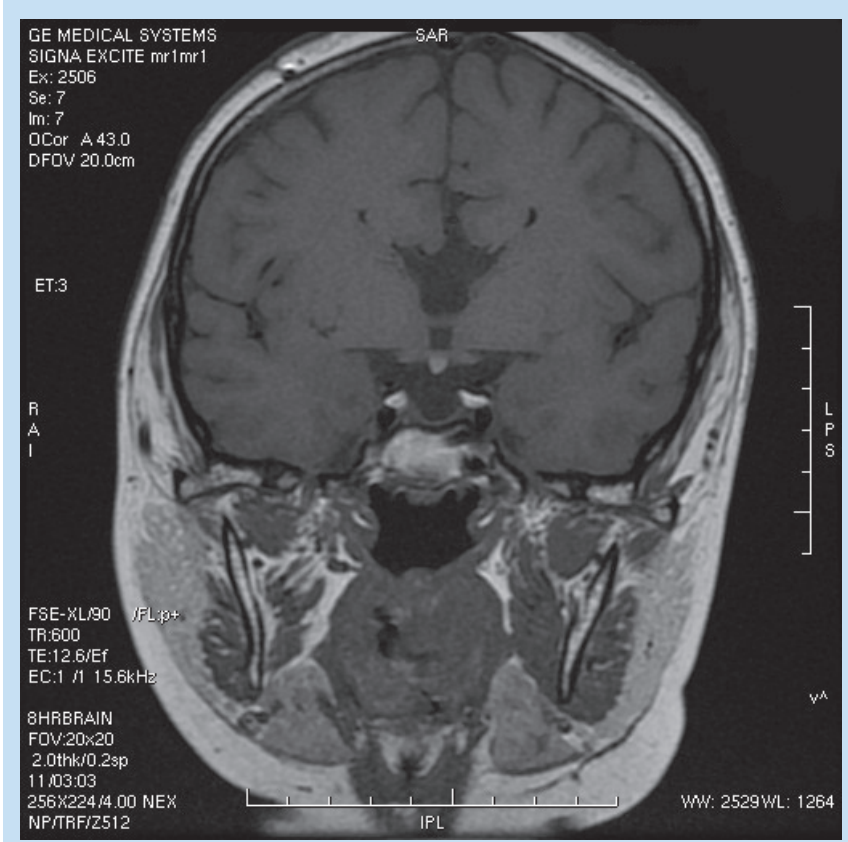

Fig. 3b. A coronal T1W MRI scan demonstrating the hyperintense central ectopic posterior pituitary, and the absent septum pellucidum. The pituitary stalk was not visualised.

She was referred for a pituitary MRI as her prolactin level was found to be high.

The MRI scan demonstrated an EPP, a hypoplastic adenohypophysis, non-visualisation of the pituitary stalk, corpus callosal agenesis, absent septum pellucidum, small calibre optic nerves, and periventricular grey matter heterotopia.

The co-existence of an EPP, periventricular grey matter heterotopia, corpus callosal agenesis and septo-optic dysplasia has been previously 


\section{CASE SERIES}

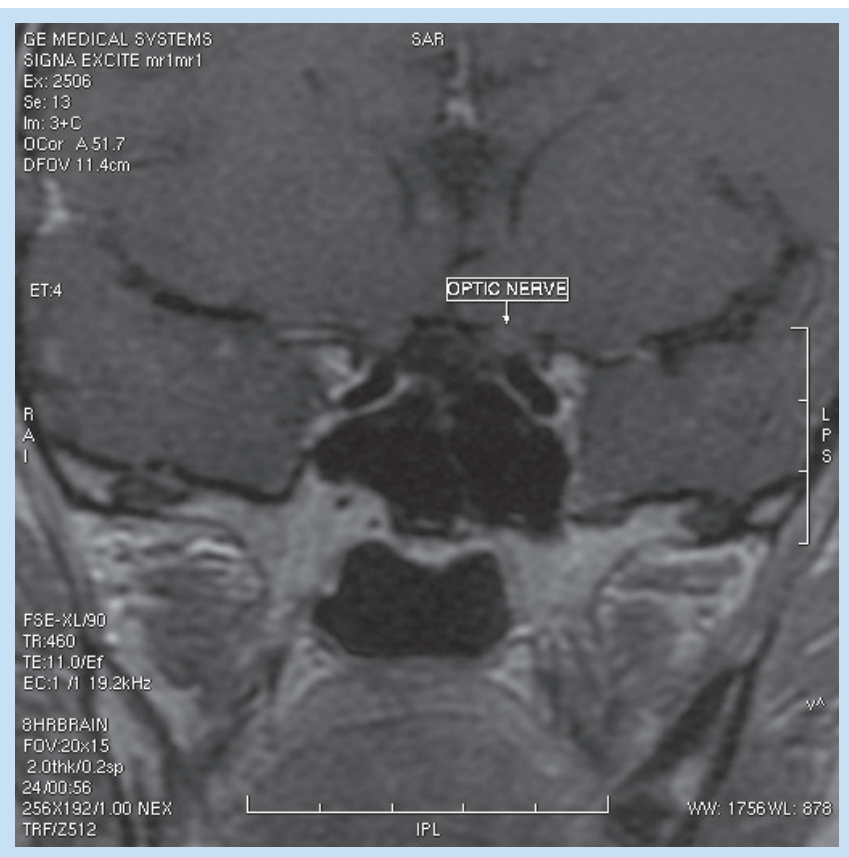

Fig. 3c. A coronal post-contrast zoomed T1W MRI scan demonstrating the hypoplastic optic nerves. The pituitary stalk was not visualised.

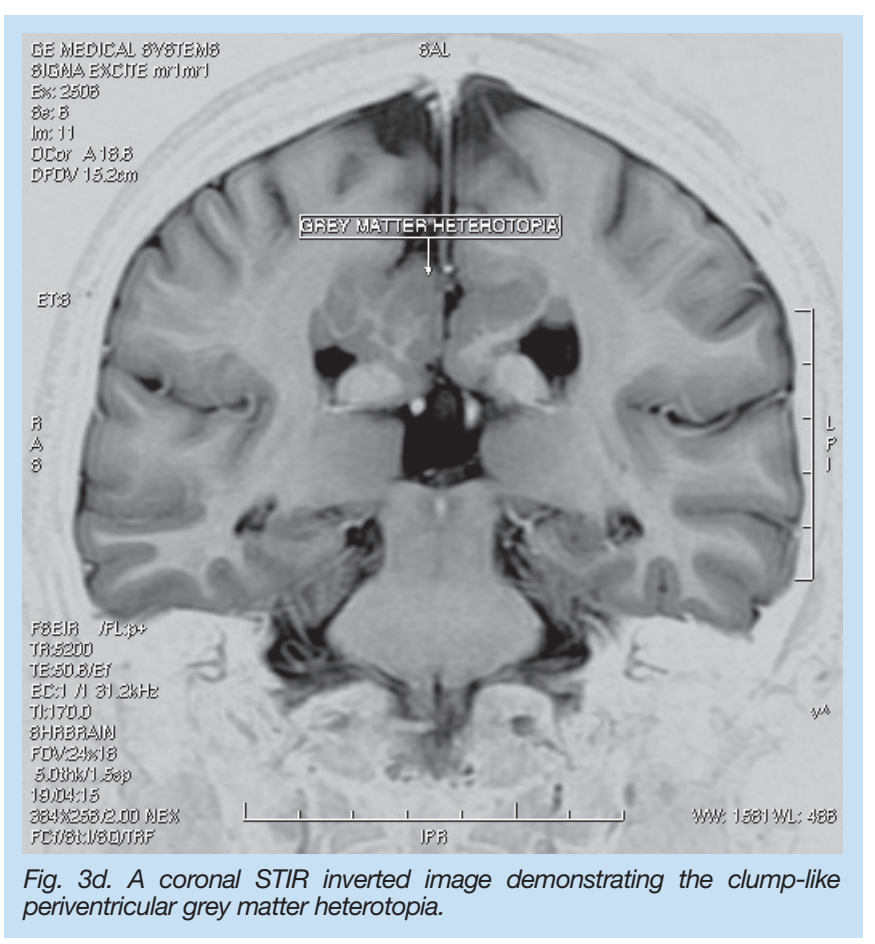

documented. ${ }^{3}$ The patient had not undergone any previous MR imaging (Figs 3 a - d).

\section{Conclusion}

The EPP should alert the radiologist to perform a precise MRI study as it is an important marker of anterior pituitary structure and function. A sagittal T1-weighted sequence is imperative, while zoomed thin slice pre- and post dynamic contrast scans of the hypothalamic-pituitary axis are necessary to fully assess the anatomy, to plan further investigations and for future management. Coronal imaging is helpful, as seen above, for evaluation of the optic nerves and septum pellucidum. A coronal STIR or T1 FLAIR assists in delineating the grey matter heterotopia better. The use of contrast is often necessary to identify and characterise the stalk and adenohypophysis. ${ }^{2}$

The following associations need to be looked for when assessing patients with an EPP.

An ectopic posterior lobe of the pituitary with a hypoplastic or absent pituitary stalk may be observed in patients with hypopituitarism, and is a specific marker of permanent GH defiency.,

An EPP may be part of the spectrum associated with septo-optic dysplasia. This is possibly related to the fact that some cases of septooptic dysplasia are caused by mutations of the HESX1 gene linked to EPP. $^{3}$

The co-existence of an EPP and periventricular grey matter heterotopia has also been linked to the HESX1 gene. ${ }^{3}$

It is also necessary to differentiate a stalk lipoma from an EPP.

Rarely, a normal pituitary MR study may be encountered in subjects with severe GH deficiency where there is a genetic origin for the disease. $^{2,5}$

\section{Acknowledgements}

Thanks to Dr Aadil Ahmed, Chris Hani Baragwanath Hospital.

1. Patkar D, Patankar T, Krishnan A, Prasad S, Shah J, Limdi J. MR imaging in children with ectopic pituitary gland and anterior hypopituitarism. J Postgrad Med 1999; 45: 81-83.

2. Chen S, Leger J, Garel C, Hassan M, Czernichow P. Growth hormone deficiency with ectopic neurohypophysis: anatomical variations and relationship between the visibility of the pituitary stalk asserted by magnetic resonance imaging and anterior pituitary function. J Clin Endocrinol Metab 1999; 84: 2408-2413.

3. Mitchell LA, Thomas PQ, Zacharin MR, Scheffer IE. Ectopic posterior pituitary lobe and periventricular heterotopia: Cerebral malformations with the same underlying mechanism? Am J Neuroradiol 2002; 23: $1475-1481$

4. Argyropoulou MI, Kiortsis DN. MRI of the hypothalamic-pituitary axis in children. Pediatr Radiol 2005; 35:1045-1055.

5. Maghnie M, Ghirardello S, Genovese E. Magnetic resonance imaging of the hypothalamic-pituitary unit in children suspected of hypopituitarism: who, how and when to investigate. J Endocrinol Invest 2004; 27: 496-509. 